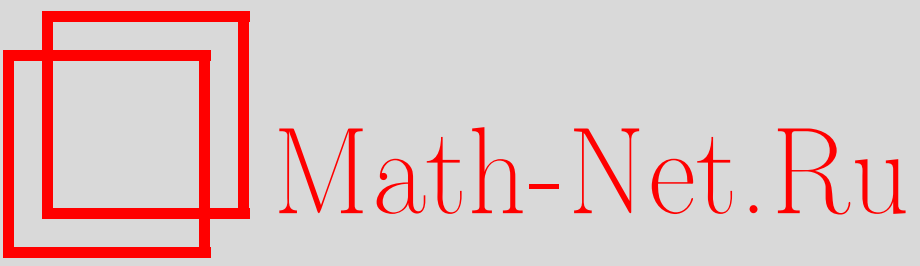

И. Г. Низовцева, Д. В. Александров, Нестационарная кристаллизация воды с двухфазной зоной при турбулентных и нетурбулентных граничных условиях, Вестн. Сам. гос. техн. ун-та. Сер. Физ.-мат. науки, 2010, выпуск 1(), 133-142

DOI: https://doi.org/10.14498/vsgtu785

Использование Общероссийского математического портала Math-Net.Ru подразумевает, что вы прочитали и согласны с пользовательским соглашением

http: //www. mathnet.ru/rus/agreement

Параметры загрузки:

IP : 54.80 .73 .141

26 апреля 2023 г., 10:32:14 
УДК 536.421.4

\title{
НЕСТАЦИОНАРНАЯ КРИСТАЛЛИЗАЦИЯ ВОДЫ С ДВУХФАЗНОЙ ЗОНОЙ ПРИ ТУРБУЛЕНТНЫХ И НЕТУРБУЛЕНТНЫХ ГРАНИЧНЫХ УСЛОВИЯХ
}

\author{
И. Г. Низовцева, Д. В. Александров
}

Уральский государственный университет им. А. М. Горького, 620083, Екатеринбург, пр-т Ленина, 51.

E-mails: nizovtseva.irina@gmail.com, dmitri.alexandrov@usu.ru

\begin{abstract}
Развита математическая модель проиессов затвердевания от охлаждаемой по произвольному закону границы в присутствии двухфазной зоны для неизотермического раствора (морской воды) в отсутствие и при наличии турбулизаиии жидкости на границе между двухфазной зоной и жидкой фазой системы. Определены распределения температуры, кониентрации примеси и доли твёрдой фазы во всех регионах протекания прочесса, найден закон движения границы «твёрдая фаза - двухфазная зона». Рассмотрены два сценария прочесса: с нулевой (который описъвает затвердевание с кристаллами игольчатой формы) и отличной от нуля (который описывает затвердевание с кристаллами с затупленной формой концов) долями твёрдой фазы на границе «двухфазная зона жсидкость». Результаты развитой теории находятся в хорошем соответствии с данными наблюдений.
\end{abstract}

Ключевые слова: кристаллизачия, морские льды, тепломассоперенос, двухфазная зона.

Введение. На первый взгляд кажущаяся тривиальной проблема роста твёрдой фазы от охлаждаемой границы берет свое начало от так называемой задачи Стефана, описывающей широкий круг физических процессов [1]. Их сложное нелинейное поведение и необходимость расчета различных характеристик этих процессов, встречающихся как в геофизике, так и в металлургии, является стимулом для разработки новых математических подходов к решению. Как уже отмечалось, процессы затвердевания бинарных растворов и расплавов часто протекают в условиях присутствия зон переохлаждения, т. е. зон в жидкой фазе системы с температурой ниже температуры фазового перехода, которая может зависеть от солености воды (концентрации растворенной примеси). Будем рассматривать ситуацию, когда концентрационное переохлаждение в двухфазной зоне снимается за счет активно растущих элементов твёрдой фазы $[2,3]$ и произведем учёт течений турбулизированной жидкости вблизи растущей границы «двухфазная зона - вода». Такой учёт приведёт к изменению пограничных условий баланса тепла и массы на этой границе. Будем основываться на предположении о линейных температурных распределениях в твёрдой фазе и двухфазной зоне (это предположение часто реализуется в естественных условиях при замерзании трещин $[4,5]$ и образовании ложного дна [6]), следующее из того обстоятельства, что время релаксации температурного поля намного меньше характерного времени движения границы «двухфазная зона - жидкая фаза». Также будем считать,

Ирина Геннадъевна Низовцева, инженер-исследователь, лаб. математического моделирования физико-химических процессов в многофазных средах. Дмитрий Валеръевич Александров (д.ф.-м.н.), профессор, каф. математической физики. 
что граница «твёрдая фаза - двухфазная зона» сильно отстаёт от границы «двухфазная зона - жидкая фаза» вследствие того, что растворенная соль вытесняется растущей твёрдой фазой в области, где её концентрация становится очень высокой. Присутствие таких областей в двухфазной зоне сильно тормозит движение границы «твёрдая фаза - двухфазная зона».

1. Нетурбулентная модель кристаллизации в условиях неизотермического океана. Будем рассматривать полуограниченную область $z>0$, изначально заполненную жидкостью солёности $S_{\infty}$ и находящуюся при температуре $T_{\infty}$. Считается, что на границе между водой и атмосферой $z=0$ в начальный момент образовалась тонкая корочка льда, а сама граница поддерживается при температуре $T_{0}(t)$, которая является произвольной функцией времени $t$. Области $a(t)<z<b(t)$ и $z>b(t)$ заполнены двухфазной зоной и жидкой фазой. В соответствии с предположениями о линейности температуры в твёрдой фазе и двухфазной зоне для распределения температуры имеем (см. также работы $[4-6])$

$$
\begin{gathered}
T_{i}(z, t)=T_{0}(t)+C_{1}(t) z, \quad 0<z<a(t), \\
T_{m}(z, t)=T_{1}(t)+T_{2}(t) z, \quad a(t)<z<b(t),
\end{gathered}
$$

где $a(t)$ и $b(t)$ - границы двухфазной зоны с твёрдой фазой и жидкостью соответственно. Линейный температурный профиль (2) в двухфазной зоне должен приближенно удовлетворять уравнению теплопроводности (здесь $L_{V}-$ скрытая теплота кристаллизации):

$$
\rho_{m}(\varphi) c_{p m}(\varphi) \frac{\partial T_{m}}{\partial t}=\frac{\partial}{\partial z}\left(k_{m}(\varphi) \frac{\partial T_{m}}{\partial z}\right)+L_{V} \frac{\partial \varphi}{\partial z}, \quad a(t)<z<b(t),
$$

где коэффициент теплопроводности и произведение плотности на теплоёмкость $k_{m}$ и $\rho_{m} c_{p m}$ могут считаться линейно зависящими от доли твёрдой фазы $\varphi$ (индексы $m, i$ и $w$ соответственно обозначают характеристики двухфазной зоны, льда и воды):

$$
k_{m}(\varphi)=k_{i} \varphi+k_{w}(1-\varphi), \quad \rho_{m}(\varphi) c_{p m}(\varphi)=\rho_{i} c_{i} \varphi+\rho_{w} c_{w}(1-\varphi) .
$$

Справедливость соотношений (4) обсуждается в работах [7-9].

Уравнение (3) будет апроксимироваться линейным профилем (2), если временные и пространственные изменения доли твёрдой фазы $\varphi$ несущественны (а это выполняется в силу быстрой релаксации температурного профиля). Это свойство согласуется с полученными решениями, а также обсуждается в работах [10-12].

Уравнение диффузии соли в двухфазной зоне и уравнение линии ликвидус имеют вид

$$
\begin{aligned}
\frac{\partial}{\partial t}\left((1-\varphi) S_{m}\right) & =D_{w} \frac{\partial}{\partial z}\left((1-\varphi) \frac{\partial S_{m}}{\partial z}\right) \\
T_{m}(z, t) & =-m S_{m}(z, t)
\end{aligned}
$$

где $S_{m}$ - концентрация примеси (солёность раствора); $D_{w}$ - коэффициент диффузии соли; $m$ - наклон линии ликвидус, определяемый из фазовой диаграммы. 
Граничные условия, выполняемые на подвижных границах $a(t)$ и $b(t)$, записываются в следующем виде (см, например, $[13,14])$ :

$$
\begin{gathered}
\varphi=\varphi_{a}, \quad T_{i}=T_{m}, \quad z=a(t), \\
L_{V}\left(1-\varphi_{a}\right) \frac{d a}{d t}=k_{i} \frac{\partial T_{i}}{\partial z}-k_{m}\left(\varphi_{a}\right) \frac{\partial T_{m}}{\partial z}, \quad z=a(t), \\
S_{m}\left(1-\varphi_{a}\right) \frac{d a}{d t}=-D_{w}\left(1-\varphi_{a}\right) \frac{\partial S_{m}}{\partial z}, \quad z=a(t), \\
\varphi=\varphi_{b}, \quad T_{m}=T_{b}, \quad z=b(t), \\
L_{V} \varphi_{b} \frac{d b}{d t}=k_{m}\left(\varphi_{b}\right) \frac{\partial T_{m}}{\partial z}-k_{w} \frac{\partial T_{w}}{\partial z}, \quad z=b(t), \\
S_{b} \varphi_{b} \frac{d b}{d t}=D_{w}\left(1-\varphi_{b}\right) \frac{\partial S_{m}}{\partial z}-D_{w} \frac{\partial S_{w}}{\partial z}, \quad z=b(t), \\
\frac{\partial T_{w}}{\partial z}=-m \frac{\partial S_{w}}{\partial z}, \quad z=b(t) .
\end{gathered}
$$

Напомним, что условия (7) и (10) отражают непрерывность температуры, однако доля твёрдой фазы $\varphi$ может и не быть непрерывной [13]. Граничные условия (8), (9) и (11), (12) представляют собой условия баланса тепла и массы на движущихся границах. Условие (13) показывает, что концентрационное переохлаждение скомпенсировано в граничной точке двухфазной зоны [13].

В области, занятой жидкостью, выполняются следующие уравнения теплопроводности и диффузии:

$$
\frac{\partial T_{w}}{\partial t}=a_{w} \frac{\partial^{2} T_{w}}{\partial z^{2}}, \quad \frac{\partial S_{w}}{\partial t}=D_{w} \frac{\partial^{2} S_{w}}{\partial z^{2}}, \quad z>b(t),
$$

где $a_{w}=k_{w} /\left(\rho_{w} c_{p w}\right)$.

Модель (1)-(14) полностью описывает динамику процесса затвердевания в нетурбулентных условиях, т.е. в случае, когда течение жидкости в океане не вносит существенных изменений в потоки тепла и массы на границе «двухфазная зона - жидкая фаза» и справедливы пограничные условия (11) и $(12)$.

Перейдём теперь к вопросу построения аналитических решений сформулированной модели. В случае слабых отклонений закона движения границы $b(t)$ от зависимости, пропорциональной корню из времени [5], решения уравнений (14) могут быть аппроксимированы следующими выражениями:

$$
\begin{gathered}
T_{w}(z, t)=T_{\infty}+\frac{\left(T_{b}-T_{\infty}\right) \operatorname{erfc}\left(z / \sqrt{4 a_{w} t}\right)}{\operatorname{erfc}\left(b(t) / \sqrt{4 a_{w} t}\right)}, \\
S_{w}(z, t)=S_{\infty}+\frac{\left(S_{b}-S_{\infty}\right) \operatorname{erfc}\left(z / \sqrt{4 D_{w} t}\right)}{\operatorname{erfc}\left(b(t) / \sqrt{4 D_{w} t}\right)},
\end{gathered}
$$

где $S_{b}=-T_{b} / m$, а $T_{b}$ на поверхности $b(t)$ находится подстановкой распределений (15) и (16) в условие (13) в зависимости от координаты $b$ и времени $t$ :

$$
T_{b}=\frac{T_{\infty} f_{1}+m S_{\infty} f_{2}}{f_{1}-f_{2}},
$$


где

$f_{1}=\sqrt{\frac{D_{w}}{a_{w}}} \exp \left(-\frac{b^{2}(t)}{4 a_{w} t}\right) \operatorname{erfc}\left(\frac{b(t)}{\sqrt{4 D_{w} t}}\right), \quad f_{2}=\exp \left(-\frac{b^{2}(t)}{4 D_{w} t}\right) \operatorname{erfc}\left(\frac{b(t)}{\sqrt{4 a_{w} t}}\right)$.

Для интегрирования уравнения диффузии (5) выразим $T_{2}(t) \partial \varphi / \partial z$ из уравнения (3) (чтобы сделать это, для простоты пренебрегаем левой частью уравнения (3) в силу того обстоятельства, что время релаксации поля температуры на несколько порядков меньше соответствующего времени для поля концентрации растворенной соли). Итак, из (2) и (3) имеем

$$
T_{2}(t) \frac{\partial \varphi}{\partial z}=-\frac{L_{V}}{k_{i}-k_{w}} \frac{\partial \varphi}{\partial t} .
$$

Теперь, подставляя распределение (2) и найденную зависимость в уравнение (5), интегрируем уравнение диффузии и записываем распределение доли твёрдой фазы в двухфазной зоне:

$$
\varphi(z, t)=\frac{T_{m}(z, t)-T_{b}+\left(T_{b}+T_{c}\right) \varphi_{b}}{T_{m}(z, t)+T_{c}}, \quad T_{c}=\frac{D_{w} L_{V}}{k_{i}-k_{w}}
$$

где $T_{b}\left(1-\varphi_{b}\right)-T_{c} \varphi_{b}$ слабо зависит от времени $t$. Подставляя $z=a(t)$ в $(18)$, находим долю твёрдой фазы на границе $a(t)$ :

$$
\varphi_{a}=\frac{T_{1}+a T_{2}-T_{b}+\left(T_{b}+T_{c}\right) \varphi_{b}}{T_{1}+a T_{2}+T_{c}}
$$

Подстановка выражений (1), (2) и (6) в условия (7), (9) и (10) позволяет выразить коэффициенты $T_{1}(t), T_{2}(t)$ и $C_{1}(t)$ :

$$
\begin{gathered}
T_{1}(t)=T_{b}(t)-b(t) T_{2}(t), \quad T_{2}(t)=-T_{b}(t) \frac{d a}{d t}\left(D_{w}+\left(a(t)-b(t) \frac{d a}{d t}\right)\right)^{-1}, \\
C_{1}(t)=\frac{(a(t)-b(t)) T_{2}(t)+T_{b}(t)-T_{0}(t)}{a(t)} .
\end{gathered}
$$

Теперь, комбинируя выражения (8), (11), (12), (20) и (21), получаем три соотношения для нахождения положений границ $a(t), b(t)$ и доли твёрдой фазы $\varphi_{b}$ :

$$
\begin{aligned}
L_{V}\left(1-\varphi_{a}\right) a \frac{d a}{d t} & =k_{i}\left(T_{b}-T_{0}\right)+T_{b} \frac{d a}{d t} \frac{\left(k_{i} \varphi_{a}+k_{w}\left(1-\varphi_{a}\right)\right) a-k_{i}(a-b)}{D_{w}+(a-b) d a / d t}, \\
L_{V} \varphi_{b} \frac{d b}{d t} & =-\frac{k_{i} \varphi_{b}+k_{w}\left(1-\varphi_{b}\right)}{D_{w}+(a-b) d a / d t} T_{b} \frac{d a}{d t}-k_{w}\left(T_{b}-T_{\infty}\right) F_{1}(b, t), \\
T_{b} \varphi_{b} \frac{d b}{d t} & =-\frac{D_{w}\left(1-\varphi_{b}\right)}{D_{w}+(a-b) d a / d t} T_{b} \frac{d a}{d t}-D_{w}\left(T_{b}+m S_{\infty}\right) F_{2}(b, t),
\end{aligned}
$$

где

$$
F_{1}=-\frac{2}{\sqrt{4 \pi a_{w} t}} \frac{\exp \left[-b^{2} /\left(4 a_{w} t\right)\right]}{\operatorname{erfc}\left[b / \sqrt{4 a_{w} t}\right]}, \quad F_{2}=-\frac{2}{\sqrt{4 \pi D_{w} t}} \frac{\exp \left[-b^{2} /\left(4 D_{w} t\right)\right]}{\operatorname{erfc}\left[b / \sqrt{4 D_{w} t}\right]} .
$$


Выражение (23) можно представить в виде

$$
\varphi_{b}=-k_{w} \frac{T_{b} d a / d t+\left(T_{b}-T_{\infty}\right) F_{1}\left(D_{w}+(a-b) d a / d t\right)}{L_{V}\left(D_{w}+(a-b) d a / d t\right) d b / d t+\left(k_{i}-k_{w}\right) T_{b} d a / d t} .
$$

Специально подчеркнём, что граничные условия (23) и (24) становятся идентичными при $\varphi_{b}=0$. Эта ситуация соответствует спокойному установившемуся режиму затвердевания (см., например, лабораторные эксперименты [15]). В этой ситуации твёрдая фаза вблизи границы $b(t)$ растет в виде иголок (для автомодельного процесса затвердевания эта ситуация была разобрана в работе [16]). Другая ситуация $\left(\varphi_{b} \neq 0\right)$ может наблюдаться в случае размывания жидкостью игольчатой формы кристаллов (например, в случае турбулентного течения жидкости [17]). Тем не менее рассмотрим обе ситуации затвердевания при $\varphi_{b}=0$ и $\varphi_{b} \neq 0$ в отсутствии турбулентности.

Рассмотрим сначала случай $\varphi_{b}=0$. Из выражений $(17),(19),(25)$ и условия $f_{1} F_{2}=f_{2} F_{1}$ имеем

$$
\begin{gathered}
\varphi_{a}=\frac{T_{b}(b-a) d a / d t}{\left(T_{b}+T_{c}\right) D_{w}+T_{c}(a-b) d a / d t} \\
\frac{d a}{d t}=-\frac{\left(T_{\infty}+m S_{\infty}\right) D_{w} f_{1} F_{2}}{T_{\infty} f_{1}+m S_{\infty} f_{2}+\left(T_{\infty}+m S_{\infty}\right)(a-b) f_{1} F_{2}} .
\end{gathered}
$$

Далее, учитывая, что $a \ll b$ (процесс слабо зависит от положения границы $a)$, из выражений $(22)$ и (26) будем иметь

$$
\frac{d a}{d t}=\frac{\left(T_{0}-T_{b}\right) D_{w}}{b T_{0}}
$$

Приравнивая теперь левые части выражений (27) и (28), получаем трансцендентное уравнение для границы $b(t)$ :

$$
\frac{T_{0}-T_{b}}{b(t) T_{0}}=-\frac{\left(T_{\infty}+m S_{\infty}\right) f_{1} F_{2}}{T_{\infty} f_{1}+m S_{\infty} f_{2}-\left(T_{\infty}+m S_{\infty}\right) b f_{1} F_{2}} .
$$

Положение границы $a(t)$ находится интегрированием выражения (28). Здесь $T_{0}=T_{0}(t), T_{b}=T_{b}(t, b(t)), f_{1}=f_{1}(t, b(t)), f_{2}=f_{2}(t, b(t)), F_{1}=F_{1}(t, b(t))$, $F_{2}=F_{2}(t, b(t)), T_{\infty}=T_{\infty}(t)$ и $S_{\infty}=S_{\infty}(t)$.

Рассмотрим теперь случай $\varphi_{b} \neq 0$. Учитывая снова, что $a \ll b$, и пренебрегая $a$ в (22), получаем (28). Далее, выражая $\varphi_{b}$ из (23) и (24) и приравнивая результат, получаем громоздкое дифференциальное уравнение первого порядка, определяющее динамику границы $b(t)$. Опуская ряд математических преобразований, запишем его окончательный вид в форме задачи Коши:

$$
\frac{d b}{d t}=\frac{D_{w} k_{i}\left[T_{\infty} f_{1}+m S_{\infty} f_{2}-T_{0}\left(f_{1}-f_{2}\right)\right]}{b(t)\left[D_{w} L_{V}\left(f_{1}-f_{2}\right)-k_{w}\left(T_{\infty} f_{1}+m S_{\infty} f_{2}\right)\right]},
$$

где $b(0)$ - известный параметр, а аргументы функций, как и ранее, зависят от $t$ и $b(t)$. Положение границы $a(t)$ находится интегрированием выражения (28). 
Граничное значение доли твёрдой фазы $\varphi_{a}$ после подстановки $T_{1}$ и $T_{2}$ из $(20)$ в (19) может быть записано в виде

$$
\varphi_{a}(t)=\frac{T_{b} b\left(1-\varphi_{b}\right) d a / d t+\left(T_{b}+T_{c}\right) \varphi_{b} D_{w}-T_{c} \varphi_{b} b d a / d t}{\left(T_{b}+T_{c}\right) D_{w}-T_{c} b d a / d t},
$$

где учтено, что $a(t) \ll b(t)$.

На рис. 1 проводится сравнение развиваемой теории с данными лабораторных экспериментов при $\varphi_{b}=0$. Для сравнения выбран именно этот случай, поскольку в экспериментах наблюдалась игольчатая форма роста твёрдой фазы.

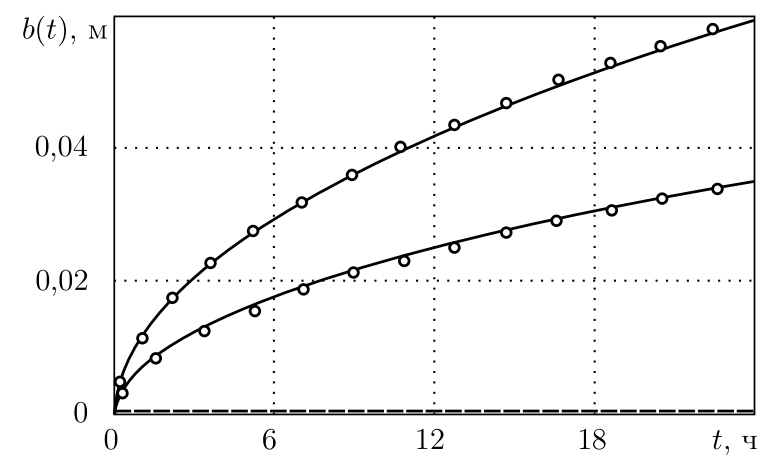

Рис. 1. Зависимость $b(t)$ : сплошные линии - расчёт по (30), значки - экспериментальные данные [13, 15], штриховая линия - расчётное положение границы $a(t) \ll b(t)$

Следующий раздел посвящен развитию теории, более приближенной к естественным условиям, где основное внимание уделяется наличию турбулентного переноса тепла и массы.

2. Турбулентная модель кристаллизации в условиях неизотермического океана. В этой ситуации процесс кристаллизации существенно зависит от турбулентного течения жидкости в океане (см., например, [18-20]). С математической точки зрения эта ситуация соответствует новым пограничным условиям на границе «двухфазная зона - океан». Заменяя тепловой и диффузионный потоки в условиях (11) и (12) турбулентными потоками в соответствии с работой [20], получим

$$
\begin{aligned}
L_{V} \phi_{b} \frac{d b}{d t} & =k_{m}\left(\phi_{b}\right) \frac{\partial T_{m}}{\partial z}-\alpha_{h} \rho_{w} c_{p w} u\left(T_{\infty}-T_{b}\right), \quad z=b(t), \\
S_{b} \varphi_{b} \frac{d b}{d t} & =D_{w}\left(1-\varphi_{b}\right) \frac{\partial S_{m}}{\partial z}-\alpha_{s} u\left(S_{\infty}-S_{b}\right), \quad z=b(t),
\end{aligned}
$$

где $\alpha_{h}$ и $\alpha_{s}$ - коэффициенты турбулентного переноса тепла и массы, $u$ - скорость трения.

Отношение коэффициентов $\alpha_{h} / \alpha_{s}$ зависит от коэффициентов теплопроводности $\left(a_{w}\right)$ и диффузии $\left(D_{w}\right)$ в воде. По данным работы [19] имеем $\alpha_{h} / \alpha_{s}=$ $=\left(a_{w} / D_{w}\right)^{n}$, где $2 / 3<n<4 / 5[21,22]$. Остальные уравнения и граничные условия модели (выражения (1)-(10)) остаются без изменений, кроме условия (13). В случае турбулентного течения жидкости это условие, вообще говоря, 
становится неприменимым, т. к. отсутствует физическая причина пропорциональности между градиентами тепла и массы.

Таким образом, в расматриваемом случае имеем следующую модель: (1)(10), (32) и (33).

Выше уже упоминалась принципиальная возможность реализации двух ситуаций кристаллизации с $\varphi_{b}=0$ и $\varphi_{b} \neq 0$. Хотя второй случай в данных условиях представляется более реалистическим и часто встречающимся в геофизике, проанализируем обе ситуации.

Рассматривая ситуацию $\varphi_{b}=0$ и считая, что $a(t) \ll b(t)$, после подстановки $d a / d t$ из (28) в (32) находим положение границы «двухфазная зона океан» :

$$
b(t)=\frac{k_{w}\left(T_{0}(t)-T_{b}(t)\right)}{\alpha_{h} \rho_{w} c_{p w} u(t)\left(T_{b}(t)-T_{\infty}(t)\right)},
$$

где $T_{b}(t)$ определяется подстановкой $\partial T_{m} / \partial z=-m\left(\partial S_{m} / \partial z\right)$ из (32) в (33). В результате получим

$$
T_{b}(t)=-m S_{b}(t)=-\frac{m S_{\infty}(t)+P T_{\infty}(t)}{1-P}, \quad P=\frac{\alpha_{h}}{\alpha_{s}} \frac{D_{w}}{a_{w}} .
$$

Положение границы «двухфазная зона - твёрдая фаза» находится интегрированием выражения (28):

$$
a(t)=a(0)+\int_{0}^{t} \frac{\left(T_{0}(t)-T_{b}(t)\right) D_{w}}{b(t) T_{0}(t)} d t .
$$

Рассмотрим теперь вторую ситуацию $\varphi_{b} \neq 0$, описывающую размывание жидкостью игольчатой формы растущих кристаллов. Учитывая соотношение (28), справедливое как для турбулентных, так и для нетурбулентных условий, и учитывая, что $a(t) \ll b(t)$, из граничных условий $(32)$ и (33) получим два выражения для доли твёрдой фазы $\varphi_{b}(t)$ :

$$
\begin{gathered}
\varphi_{b}=-\frac{k_{w}\left(T_{b}-T_{0}\right)-\alpha_{h} \rho_{w} c_{p w} u\left(T_{\infty}-T_{b}\right) b}{\left(k_{i}-k_{w}\right)\left(T_{b}-T_{0}\right)-L_{V} b d b / d t}, \\
\varphi_{b}=\frac{\alpha_{s} u\left(m S_{\infty}+T_{b}\right) b+D_{w}\left(T_{b}-T_{0}\right)}{D_{w}\left(T_{b}-T_{0}\right)+b T_{b} d b / d t} .
\end{gathered}
$$

Приравнивая теперь правые части (37) и (38), приходим к задаче Коши для определения $b(t)$ :

$$
\frac{d b}{d t}=\frac{\left(T_{b}-T_{0}\right)\left[b f_{3}+k_{i} D_{w}\left(T_{b}-T_{0}\right)\right]}{b\left[b f_{4}+\left(T_{b}-T_{0}\right)\left(D_{w} L_{V}-T_{b} k_{w}\right)\right]}
$$

где

$$
\begin{gathered}
f_{3}(t)=u(t)\left[\alpha_{s}\left(m S_{\infty}(t)+T_{b}(t)\right)\left(k_{i}-k_{w}\right)-\alpha_{h} \rho_{w} c_{p w}\left(T_{\infty}(t)-T_{b}(t)\right) D_{w}\right], \\
f_{4}(t)=u(t)\left[\alpha_{s} L_{V}\left(m S_{\infty}(t)+T_{b}(t)\right)+T_{b}(t) \alpha_{h} \rho_{w} c_{p w}\left(T_{\infty}(t)-T_{b}(t)\right)\right],
\end{gathered}
$$

a $b(0)$ - заданная величина. Положение второй границы $a(t)$, доли твёрдой фазы $\varphi_{a}$ и $\varphi_{b}$ определяются выражениями $(28),(31)$ и $(37)$, а $T_{b}$ представляет 
собой заданную температуру, в отличие от нетурбулентного сценария кристаллизации, где она определялась выражением (13). Таким образом, в турбулентном случае граничных условий получается на одно меньше, чем в нетурбулентном случае. $\mathrm{C}$ физической точки зрения температура $T_{b}$ должна полностью определяться турбулентным течением жидкости вблизи границы $b(t)$, которое, в свою очередь, зависит от многих факторов (например, от географических координат места расположения трещины и погодных условий).

Полагая скорость трения $u$ в выражении (39) нулевой и интегрируя результат, получим приближенное выражение для $b(t)$ для случая очень медленного течения воды:

$$
b(t)=\sqrt{2 k_{i} D_{w} \int_{0}^{t} \frac{T_{b}-T_{0}}{D_{w} L_{V}-T_{b} k_{w}} d t+b^{2}(0)} .
$$

Структура полученного интеграла похожа на свой аналог для случая изотермического океана $[10,11]$.

Для сравнения развиваемой теории с экспериментом снова обратимся к кристаллизации молодого льда. Рассмотрим образование такого льда по данным полевых наблюдений группы LeadEx [4] (см. также [5]). На рис. 2 и 3 демонстрируется распределение температуры в области кристаллизации на

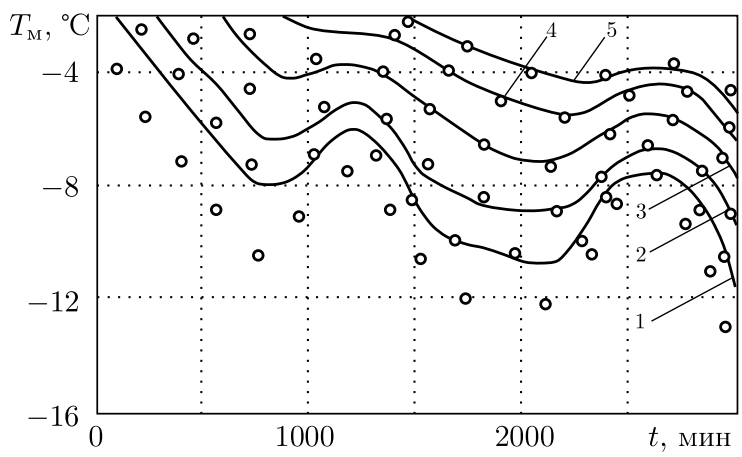

Рис. 2. Температурные профили на различных глубинах $z$ в зависимости от времени: значки - эксперимент $[4,5]$, линии - расчёт; маркеры: $1-z=0$, $2-z=2 \mathrm{~cm}, 3-z=4 \mathrm{~cm}, 4-z=6 \mathrm{~cm}, 5-z=8 \mathrm{~cm}$

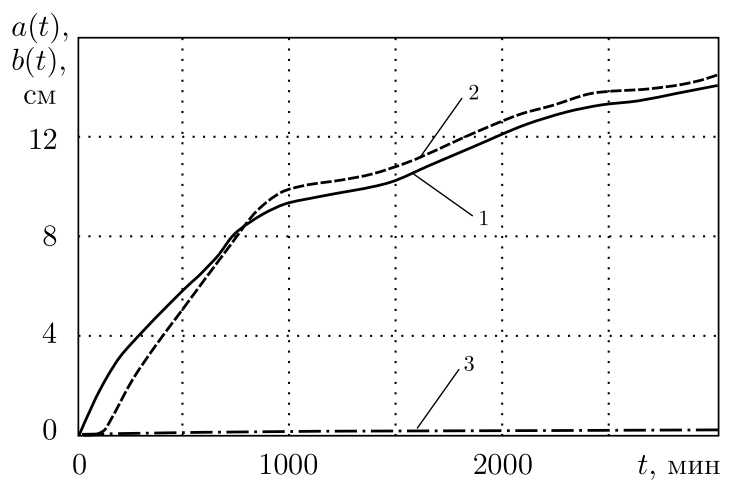

Рис. 3. Временные зависимости положений границ $a(t)$ и $b(t)$ для экспериментов $[4,5]: 1-b(t)$ (буй 5$), 2-b(t)$ (буй 6$), 3-a(t)$ (буй 5$)$ 
различных глубинах $z$ (z-глубина, отмеренная от границы «атмосфера лёд») и координаты межфазных границ в зависимости от времени в соответствии с развиваемой в настоящем разделе теорией и данными наблюдений $[4,5]$. При расчётах использовались следующие значения параметров [20]: $T_{b}=-1,97{ }^{\circ} \mathrm{C}, m=0,052{ }^{\circ} \mathrm{C} / \%$ o, $L_{V}=3,072 \cdot 10^{8}(\mathrm{BT} \cdot \mathrm{c}) / \mathrm{m}^{3}, k_{i}=$ $=2,03 \mathrm{BT} /\left(\mathrm{M} \cdot{ }^{\circ} \mathrm{C}\right), k_{w}=0,56 \mathrm{BT} /\left(\mathrm{M} \cdot{ }^{\circ} \mathrm{C}\right), \rho_{w}=1000 \mathrm{Kr} / \mathrm{m}^{3}, D_{w}=1,2 \cdot 10^{-9} \mathrm{M}^{2} / \mathrm{c}$, $c_{p w}=4187(\mathrm{BT} \cdot \mathrm{c}) /\left(\mathrm{\kappa} \Gamma \cdot{ }^{\circ} \mathrm{C}\right), T_{\infty}=-1,7^{\circ} \mathrm{C}, S_{\infty}=34,5 \%$ o $, \alpha_{s} u=0,18 \cdot 10^{-5} \mathrm{~m} / \mathrm{c}$, $\alpha_{h} / \alpha_{s}=18,75$. Кривая $1(z=0)$ на рис. 2 представляет собой атмосферную температуру на поверхности льда. На рис. 3 показаны колеблющиеся со временем законы движения границ вследствие течения жидкости в океане. Отметим, что граница $a(t)$ сильно отстает от границы $b(t)$, как это и предполагалось при построении теории. Полученные результаты находятся в хорошем соответствии с экспериментальными данными.

Заключение. В настоящей работе определены аналитические решения затвердевания бинарного раствора с двухфазной зоной в условиях турбулентного движения жидкости в океане. В ситуации, когда $\varphi_{b}=0$, определен явный вид законов движения границ двухфазной зоны. В ситуации, когда $\varphi_{b} \neq 0$, указанные законы найдены в явном виде при постоянных внешних параметpax системы. В случае, когда эти параметры изменяются со временем, в явном виде определен закон движения границы $a(t)$, а для нахождения границы $b(t)$ выписана задача Коши.

Работа выполнена при поддержке РФФИ (код проекта 08-01-00298), а также в рамках реализачии ФЦП «Научные и научно-педагогические кадры инновачионной России» на 2009-2013 годы (гос. контракт № П 819).

\section{БИБЛИОГРАФИЧЕСКИЙ СПИСОК}

1. Рубинштейн Л. И. Проблема Стефана. - Рига: Звайгзне, 1967. - 457 с.

2. Борисов В. Т. Теория двухфазной зоны металлического слитка. - М.: Металлургия, 1987. $-224 \mathrm{c}$.

3. Hills R. N., Loper D. E., Roberts P. H. The thermodynamically consistent model of a mushy zone // Q. J. Mech. Appl. Math., 1983. — Vol. 36. — P. 505-539.

4. The LeadEx Group The LeadEx experiment // Eos Trans. AGU, 1993. - Vol. 74, No. 35. P. 393-397.

5. Wettlaufer J.S., Worster M. G., Huppert H. E. Solidification of leads: Theory, experiment, and field observations // J. Geophys. Res., 2000. - Vol. 105, No. C1. - P. 1123-1134.

6. Martin S., Kauffman P. The evolution of under-ice melt ponds, or double diffusion at the freezing point // J. Fluid Mech., 1974. - Vol.64, No. 3. - P. 507-528.

7. Batchelor G. K. Transport Properties of Two-Phase Materials with Random Structure // Annu. Rev. Fluid Mech., 1974. - Vol.6. - P. 227-255.

8. Buyevich Yu. A., Alexandrov D. V. Heat Transfer in Dispersions. - New York: Begell House, Inc., 2005. - $341 \mathrm{p}$.

9. Perovich D. K., Gow A.J. A quantitative description of sea ice inclusions // J. Geophys. Res., 1996. - Vol. 101, No. C8. - P. 18,327-18,343.

10. Alexandrov D. V., Malygin A.P., Alexandrova I. V. Solidification of leads: approximate solutions of non-linear problem // Annals of Glaciology, 2006. - Vol. 44, No. 1. - P. 118122 .

11. Александров Д.В., Малыгин А.П. Аналитическое описание кристаллизации морской воды в трещинах льдов и их влияние на теплообмен между океаном и атмосферой // ДАН, 2006. - Т. 411, № 3. - С. 390-394; англ. пер.: Alexandrov D. V., Malygin A.P. Analytical description of seawater crystallization in ice fissures and their influence on heat exchange between the ocean and the atmosphere // Doklady Earth Sciences, 2006. Vol. 411, No. 2. - P. 1407-1411.

12. Alexandrov D. V., Aseev D. L., Nizovtseva I. G., Huang H.-N., Lee D. Nonlinear dynamics of directional solidification with a mushy layer. Analytic solutions of the problem // Int. J. 
Heat Mass Transfer, 2007. - Vol.50, No.17-18. - P. 3616-3623.

13. Worster M. G. Solidification of an alloy from a cooled boundary // J. Fluid Mech., 1986. Vol. 167. - P. 481-501.

14. Buyevich Yu. A., Alexandrov D. V., Mansurov V. V. Macrokinetics of crystallization. - New York: Begell House, Inc., 2001. - 183 p.

15. Huppert H. E., Worster M. G. Dynamic solidification of a binary melt // Nature, 1985. Vol. 314. - P. 703-707.

16. Alexandrov D. V., Malygin A. P. Self-similar solidification of an alloy from a cooled boundary // Int. J. Heat Mass Transfer, 2006. - Vol. 49, No. 3-4. - P. 763-769.

17. McPhee M. G. A Time-Dependent Model for Turbulent Transfer in a Stratified Oceanic Boundary Layer // J. Geophys. Res., 1987. - Vol. 92, No. C7. - P. 6977-6986.

18. McPhee M. G. The upper Ocean / In: The Geophysics of Sea Ice/ NATO ASI series. Series B, Physics; ed. N. Untersteiner. - New York: Plenum Press, 1986. - Vol. 146. P. 133-141.

19. McPhee M. G., Maykut G. A., Morison J. H. Dynamics and Thermodynamics of the Ice/Upper Ocean System in the Marginal Ice Zone of the Greenland Sea// J. Geophys. Res., 1987. - Vol. 92, No. C7. - P. 7017-7031.

20. Notz D., McPhee M. G., Worster M. G., Maykut G. A., Schlünzen K. H., Eicken H. Impact of underwater-ice evolution on Arctic summer sea ice // J. Geophys. Res., 2003. - Vol. 108, No. C7. - P. 3223

21. Owen P. R., Thomson W. R. Heat transfer across rough surfaces // J. Fluid Mech., 1963. Vol. 15, No. 3. - P. 321-334

22. Yaglom A.M., Kader B. A. Heat and mass transfer between a rough wall and turbulent fluid flow at high Reynolds and Péclet numbers // J. Fluid Mech., 1974. - Vol.62, No. 3. P. 601-623.

MSC: 80A22, 80A20, 76F40, 86A40

Поступила в редакцию $16 / \mathrm{II} / 2010$;

в окончательном варианте - 04/III/2010.

\section{NON-STATIONARY CRYSTALLIZATION OF WATER WITH A MUSHY LAYER IN THE TURBULENT AND NON-TURBULENT BOUNDARY CONDITIONS}

\section{G. Nizovtseva, D. V. Alexandrov}

Ural State University,

51, pr. Lenina, Ekaterinburg, 620083.

E-mails: nizovtseva.irina@gmail.com, dmitri.alexandrov@usu.ru

In the present work, we developed a mathematical model of the solidification processes from a cooling by an arbitrary law boundaries in the presence of mushy layer for non-isothermal solution (sea water) in the absence and presence of turbulence in the liquid at the boundary between the mushy layer and liquid phase of the system. The distribution of temperature, impurity concentration and the solid phase fraction in all regions of the process, and also the law of motion of the solid phase - mushy layer boundary were found. We consider two scenarios of the process: with no solid phase (which describes the solidification with some needle-shaped crystals) and with some (which describes the solidification of a blunt-end crystals) portion of the solid phase at the boundary of mushy layer and liquid. The results of the developed theory are in good agreement with observations.

Key words: crystallization, sea ice, heat and mass transfer, mushy layer.

Original article submitted 16/II/2010; revision submitted $04 / \mathrm{III} / 2010$.

Irina G. Nizovtseva, Engineer Researcher, Lab. of Mathematical Modeling of Physical and Chemical Processes in Multiphase Media. Dmitri V. Alexandrov (Dr. Sci (Phys. \& Math.)), Professor, Dept. of Mathematical Physics. 